\title{
Determination of the Population Pharmacokinetic Parameters of Sustained-Release and Enteric-Coated Oral Formulations, and the Suppository Formulation of Diclofenac Sodium by Simultaneous Data Fitting Using NONMEM
}

\author{
Nasir M. Idkaidek ${ }^{b}$, Gordon L. Amidon ${ }^{a, *}$, David E. Smith ${ }^{a}$, Naji M. Najib ${ }^{b}$ and Mazen M. Hassan \\ ${ }^{a}$ College of Pharmacy, The University of Michigan, Ann Arbor, MI 48109-1065, USA \\ ${ }^{\mathrm{b}}$ Department of Pharmaceutics, Faculty of Pharmacy, University of Science and Technology, Irbid, Jordan \\ ${ }^{c}$ Faculty of Pharmacy and Medical Sciences, Amman University, Amman, Jordan
}

\begin{abstract}
Data from sustained-release and enteric-coated oral formulations, and the suppository formulation of diclofenac sodium are fitted simultaneously using NONMEM $^{\circledR}$ and the general linear model, ADVAN 5. Absorption and disposition parameters, serum levels, and absorption profiles were determined. The in vivo absorption profiles were determined using the program TOPFIT ${ }^{\circledR}$. The in vivo absorption for the sustained-release formulation is slow first order and follows a flip-flop model since disposition rate constants are greater than absorption rate constants. Absorption from the enteric-coated form is essentially complete $(\geq 95 \%)$ at about $7.5 \mathrm{~h}$, while it is $95 \%$ complete at $24 \mathrm{~h}$ from the sustained-release formulation. This suggests likely absorption from the colon in the case of the sustainedrelease formulation since absorption is only $75 \%$ complete during the first $10 \mathrm{~h}$. The sustained-release relative bioavailability is $90-99 \%$. Absorption from the suppository is essentially complete at about $4.5 \mathrm{~h}$. However, the relative bioavailability of the suppository formulation is low (55\%), since defecation may remove the drug from the absorption site before complete absorption. (c) 1998 John Wiley \& Sons, Ltd.
\end{abstract}

Key words: diclofenac sodium; pharmacokinetics; suppository; sustained release; enteric coated

\section{Introduction}

Diclofenac sodium is a non-steroidal anti-inflammatory, analgesic, and antipyretic agent with a good gastrointestinal tolerability [1]. It is effective in the treatment of rheumatoid arthritis and other arthritic conditions [2]. The drug has been marketed internationally since 1973 and is currently available in oral, rectal, parenteral, and topical preparations. An oral enteric-coated tablet and eye solution are the only formulations available commercially in the United States $[3,4]$.

Diclofenac sodium is rapidly and completely absorbed following oral administration of the conventional formulation. However, the bioavailability of the unchanged drug is about 54\% [5]. Absorption in man is very rapid from oral drug solution with a $t_{\max }$ of 5-10 min after administration of a buffered aqueous solution to fasted healthy subjects [6]. In the absence of disease or other interacting drugs,

\footnotetext{
* Correspondence to: College of Pharmacy, The University of Michigan, 428 Church Street, Ann Arbor, MI 48109-1065, USA. Tel.: + 13137642440. For detailed modeling and methodology information please contact Dr Amidon.

Contract grant sponsor: Jordan University of Science and Technology; Contract grant number: FDA
}

diclofenac absorption, metabolism, and excretion do not appear to be influenced by age [7].

Sustained-release formulations offer several advantages over conventional (enteric-coated) delivery systems. These include controlling the dose release and hence decreasing the possible incidence of toxic effects, and reducing the dosing frequency and therefore improving patient compliance. However, it has been reported that sustained-release formulations are less bioavailable than conventional formulations [8]. Other studies indicated that both types of formulations have the same bioavailability [9].

Diclofenac sodium is a weak acid with a $\mathrm{p} k_{\mathrm{a}}$ of 4.0 [10] and an octanol-buffer ( $\mathrm{pH}$ 7.4) partition coefficient of 13.4 [1]. It is poorly soluble in acidic medium, $0.003 \mathrm{mg} \mathrm{mL}^{-1}$ in simulated gastric fluid, and highly soluble in basic medium, $13 \mathrm{mg} \mathrm{mL}^{-1}$ in simulated intestinal fluid, suggesting that the $\mathrm{pH}$ affects the solubility and absorption of diclofenac sodium [3]. In addition, buffering or increasing the fluid intake volume will increase its absorption, which may be due to $\mathrm{pH}$-dependent solubility of the drug or the effect of gastric content volume on gastric emptying [3]. It has been reported that for a sustained-release formulation, pellets (multiple units) are superior to matrix tablets (single units) 
in the context of intestinal passage because they allow better distribution in the intestine with reduced risk of local reactions [11]. Another study in animals showed that a diclofenac sodium suppository has a similar bioavailability as an oral formulation with an irritative effect on rectal mucosa [12].

To date, no detailed data analysis has been performed on diclofenac sodium on a large scale to have accurate inferences about its population pharmacokinetics. Therefore, the aim of this research is to determine the population pharmacokinetics of diclofenac sodium by simultaneous data fitting of three different drug formulations, including sustained-release and enteric-coated oral formulations, and the suppository formulation. Another aim is to determined the relative bioavailabilities of the sustained-release and the suppository formulations as compared to the enteric-coated conventional formulation and hence their suitability as drug formulations.

\section{Materials and Methods}

\section{Experimental Design}

Two separate studies were performed in the Jordan University of Science and Technology, Irbid, Jordan. All subjects were healthy male non-smokers with no history of major diseases. Volunteers were given single doses of either the sustained-release or the enteric-coated formulation of diclofenac sodium, Inflaban ${ }^{\circledR}$, with $250 \mathrm{~mL}$ water after an overnight fast of at least $10 \mathrm{~h} \mathrm{[13].} \mathrm{Food} \mathrm{and} \mathrm{bev-}$ erages were withheld for $4 \mathrm{~h}$ after the administration of the dose [13]. A third separate study on the diclofenac sodium $100 \mathrm{mg}$ suppository, Infl$\mathrm{aban}^{\circledR}$, was performed in Amman Islamic Hospital, Amman, Jordan. Volunteers were asked to administer the drug after overnight fasting. Food was served at $0.5,5$, and $11 \mathrm{~h}$ after dose administration. All the volunteers were asked to abstain from taking any medication for at least 1 week prior to the study [14].

The enteric-coated formulation study consisted of 23 subjects who were given a $(2 \times 50 \mathrm{mg})$ single oral dose of diclofenac sodium as entericcoated tablets. Serum concentrations were measured at $0,0.5,1,1.5,2,2.5,3,3.5,4,5,6,8$, and $10 \mathrm{~h}$ after dose administration [16]. The sustained-release formulation study consisted of 31 subjects who were given a $100 \mathrm{mg}$ oral dose of diclofenac sodium as film-coated matrix tablets. Serum concentrations were measured at $0,0.5,1$, $1.5,2,2.5,3,3.5,4,5,6,8,10,12$, and $24 \mathrm{~h}$ after dose administration [16]. The suppository formulation of $100 \mathrm{mg}$ drug was given to 30 subjects. Serum concentrations were measured at $0,0.25$,
$0.5,0.75,1,1.5,2,2.5,3,3.5,4,5,8$, and $10 \mathrm{~h}$ after administration [16].

\section{Assay}

Approximately $10 \mathrm{~mL}$ of venous blood samples were drawn into vacutainers. One sample was taken before dosing and the other samples were taken after dosing at timed intervals. After centrifugation at $3000 \mathrm{rpm}$ for $15 \mathrm{~min}$, serum samples were frozen at $-20^{\circ} \mathrm{C}$ until the time of analysis, which was at most 2 weeks $[13,14]$.

Serum samples were assayed for diclofenac sodium using a sensitive and specific high-performance liquid chromatographic procedure [15]. In brief, the assay involves protein precipitation of the serum samples with acetonitrile, followed by elution from a $5 \mu \mathrm{m} \mathrm{C}-8$ reversed phase column with a mobile phase consisting of acetonitrilewater $(50: 50, \mathrm{v} / \mathrm{v})$ adjusted to $\mathrm{pH} 3.3$ with glacial acetic acid, at a flow rate of $2 \mathrm{~mL} \min ^{-1}$, with ultra-violet detection at $280 \mathrm{~nm}$. Quantitation was performed by the measurement of the peak-height ratio of diclofenac sodium to the internal standard flufenamic acid. The limit of detection was $20 \mathrm{ng}$ $\mathrm{mL}^{-1}$ with a concentration range of $0.02-7.0 \mu \mathrm{g}$ $\mathrm{mL}^{-1}$.

\section{Model Building}

One-, two-, and three-compartment models were evaluated in each individual using TOPFIT. Onecompartment and three-compartment models did not fit the data adequately for all individuals. However, two-compartment model fitting was much better and fitted the data for all individuals. The criteria used for our decision involved the following: examining the fitted curves, examining the improvement in statistical tests provided by TOPFIT (i.e., Akaike test, Schwarz test and Imbimbo test in addition to correlation coefficient of the fitted curves), and examining the improvement in relative residuals $v s$. data points plots. We found that the two-compartment model was the only model that adequately fit the data for all individuals and for all formulations, and that is what is needed for simultaneous data fitting with NONMEM. In contrast, one-compartment and three-compartment models were not adequate.

Models having one lag time for one depot did not fit all the data points in each individual, and hence two lag times for two depots were utilized. This approach seemed feasible based on drug release from the dosage form and/or physiological dependence of absorption upon gastric emptying or intestinal motility. However, NONMEM still has the option of assigning an essentially zero lagtime and 


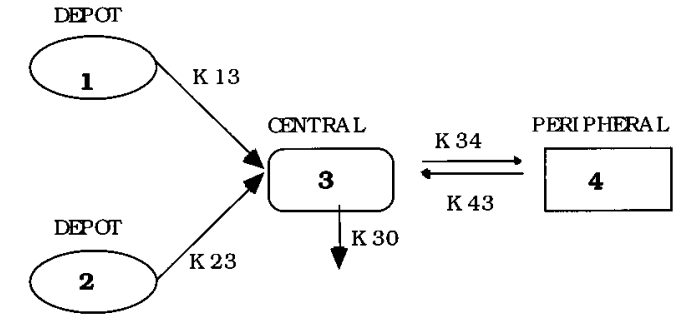

Figure 1. The pharmacokinetic model for simultaneous data fitting

zero fraction absorbed values for the first depot compartment and, hence, that individual will have essentially one depot.

This model evolution was supported by NONMEM, in which the two-compartment model gave a better goodness of fit statistic (minimum value of objective function, $\mathrm{MVOF}=11690, l_{f}$ ) than the one-compartment model $\left(\mathrm{MVOF}=11860, l_{r}\right)$. This decrease was statistically significant using the Likelihood statistic [17].

$$
\begin{aligned}
{\left[\left(C^{2}\right)=\left(l_{r}-l_{f}\right)\right.} & =170] \gg\left[q F_{0.05}(q, n-p)\right. \\
& =q F_{0.05}(17,942-24) \\
& \left.=q F_{0.05}(15, \infty)=31.11\right]
\end{aligned}
$$

and also $\gg\left[\chi_{0.05}^{2}(q)=27.6\right]$

For the three-compartment model, no convergence occurred in NONMEM due to numerical difficulties.

\section{Data Analysis}

The simultaneous data fitting of the enteric-coated formulation, the sustained-release formulation, and the suppository formulation, was performed using the NONMEM (double precision, version IV) with the NM TRAN preprocessor running on a Silicon Graphics minicomputer. The model giving the best fit of the data were a two-compartment model with two depot compartments, Figure 1 (general linear model; ADVAN 5 subroutine), with nine basic parameters, four of which are common to all forms while others vary with each form, and four inter-individual variabilities form which $K_{23}$ varies between two forms as appears in Table 1. All inter-individual variabilities are modelled according to a combined additive and proportional error model. NONMEM has the ability to fit large numbers of individual data (942 data observations from 84 individuals in this study) even though the three studies were independent [17]. The pharmacokinetic model used for simultaneous data fitting consists of two depot compartments, one central, and one peripheral compartment. The three formulations have the same disposition compartments (3 and 4) and parameters. However, they have different absorp-

\begin{tabular}{|c|c|c|c|}
\hline Parameter & Sustained & Enteric coated & Suppository \\
\hline$K_{13}\left(\mathrm{~h}^{-1}\right)$ & $\begin{array}{l}0.234 \\
(0.065)^{\mathrm{a}}\end{array}$ & $\begin{array}{l}0.437 \\
(0.095)\end{array}$ & $\begin{array}{l}2.01 \\
(0.78)\end{array}$ \\
\hline$K_{23}\left(\mathrm{~h}^{-1}\right)$ & $\begin{array}{l}0.106 \\
(0.0265)\end{array}$ & $\begin{array}{l}0.509 \\
(0.0723)\end{array}$ & $\begin{array}{l}0.667 \\
(0.0593)\end{array}$ \\
\hline$K_{34}\left(\mathrm{~h}^{-1}\right)$ & $\begin{array}{l}5.36 \\
(1.68)\end{array}$ & $\begin{array}{l}5.36 \\
(1.68)\end{array}$ & $\begin{array}{l}5.36 \\
(1.68)\end{array}$ \\
\hline$K_{43}\left(\mathrm{~h}^{-1}\right)$ & $\begin{array}{l}0.70 \\
(0.088)\end{array}$ & $\begin{array}{l}0.70 \\
(0.088)\end{array}$ & $\begin{array}{l}0.70 \\
(0.088)\end{array}$ \\
\hline$K_{30}\left(\mathrm{~h}^{-1}\right)$ & $\begin{array}{l}7.20 \\
(1.99)\end{array}$ & $\begin{array}{l}7.20 \\
(1.99)\end{array}$ & $\begin{array}{l}7.20 \\
(1.99)\end{array}$ \\
\hline$V_{\text {central }} / f(\mathrm{~L})$ & $\begin{array}{l}4.70 \\
(0.588)\end{array}$ & $\begin{array}{l}4.70 \\
(0.588)\end{array}$ & $\begin{array}{l}4.70 \\
(0.588)\end{array}$ \\
\hline Lag time $_{1}(\mathrm{~h})$ & $\left(2.73 \times 10^{-9}\right)$ & $\begin{array}{l}0.30 \\
\left(2.45 \times 10^{-10}\right)\end{array}$ & $\left(1.71 \times 10^{-9}\right)$ \\
\hline Lag time $_{2}(\mathrm{~h})$ & $\begin{array}{l}2.77 \\
(0.156)\end{array}$ & $\begin{array}{l}1.91 \\
(0.033)\end{array}$ & $\begin{array}{l}0.251 \\
\left(7.79 \times 10^{-7}\right)\end{array}$ \\
\hline Fraction absorbed from depot 1 (A1) & $\begin{array}{l}0.58 \\
(0.14)\end{array}$ & $\begin{array}{l}0.568 \\
(0.18)\end{array}$ & $\begin{array}{l}0.394 \\
(0.095)\end{array}$ \\
\hline \multicolumn{4}{|l|}{ Inter-individual variability } \\
\hline$K_{23}$ & $-^{\mathrm{b}}$ & $\begin{array}{l}3.53 \\
(2.34)\end{array}$ & $\begin{array}{l}0.72 \\
(0.0835)\end{array}$ \\
\hline$K_{34}$ & $\begin{array}{l}0.172 \\
(0.715)\end{array}$ & $\begin{array}{l}0.172 \\
(0.715)\end{array}$ & $\begin{array}{l}0.172 \\
(0.715)\end{array}$ \\
\hline Lag time $_{1}$ & - & $\begin{array}{l}37.12 \\
(53.4)\end{array}$ & - \\
\hline$A_{1}$ & - & - & $\begin{array}{l}14.51 \\
(16.1)\end{array}$ \\
\hline $\begin{array}{l}\text { Intra-individual variability } \\
\text { Residuals }\end{array}$ & 0.205 & 0.071 & 0.171 \\
\hline
\end{tabular}
tion parameters as shown in Table 1.

Table 1. NONMEM pharmacokinetic results

${ }^{a}$ Values within parenthesis are S.E.M.

${ }^{\mathrm{b}}$ Variability was not modelled. 
Table 2. Derived pharmacokinetic parameters

\begin{tabular}{llll}
\hline Parameter & Sustained & $\begin{array}{l}\text { Enteric } \\
\text { Coated }\end{array}$ & Suppository \\
\hline $\mathrm{AUC}_{0-\infty}{ }^{\mathrm{a}}$ & $6404,7065^{\mathrm{b}}$ & 7134 & 3955 \\
$\quad\left(\mathrm{ng} \mathrm{h} \mathrm{mL}^{-1}\right)$ & & & \\
$\mathrm{Cl} / f\left(\mathrm{~mL} \mathrm{~min}^{-1}\right)^{\mathrm{c}}$ & 260,236 & 234 & 421 \\
$V_{2} / f(\mathrm{~L})^{\mathrm{d}}$ & 36 & 36 & 36 \\
$V_{\mathrm{d}} / f(\mathrm{~L})^{\mathrm{e}}$ & 40.7 & 40.7 & 40.7 \\
$\mathrm{MAT}(\mathrm{h})^{\mathrm{f}}$ & 6.99 & 3.14 & 1.26 \\
$\beta\left(\mathrm{h}^{-1}\right)^{\mathrm{g}}$ & 0.392 & 0.392 & 0.392 \\
$\alpha\left(\mathrm{h}^{-1}\right)^{\mathrm{g}}$ & 12.868 & 12.868 & 12.868 \\
$t_{1 / 2} \beta(\mathrm{h})^{\mathrm{h}}$ & 1.768 & 1.768 & 1.768 \\
$t_{1 / 2} \operatorname{depot} 1(\mathrm{~h})^{\mathrm{h}}$ & 2.962 & 1.586 & 0.345 \\
$t_{1 / 2} \operatorname{depot} 2(\mathrm{~h})^{\mathrm{h}}$ & 6.54 & 1.361 & 1.04 \\
$\left.C_{\text {max }}(\mathrm{ng} \mathrm{mL})^{-1}\right)^{\mathrm{i}}$ & 582 & 1810 & 2050 \\
$t_{\text {max }}(\mathrm{h})^{\mathrm{i}}$ & 3 & 2 & 0.5 \\
$f_{\text {relative }}(\%)$ & 90,99 & 100 & 55 \\
$A_{2}{ }^{\mathrm{j}}$ & 0.370 & 0.432 & 0.606 \\
\hline
\end{tabular}

a Calculated using the linear and logarithmic trapezoidal rules for ascending and descending parts of the curve, respectively.

${ }^{\mathrm{b}} \mathrm{AUC}_{24-\infty}$ for the sustained release is calculated first using $\beta$ assuming no more absorption is taking place and hence elimination is rate limiting in the serum profile after $24 \mathrm{~h}$, then using $K_{23}$ assuming the absorption is still occurring and is hence the rate limiting step. This was done since no samples were taken after $24 \mathrm{~h}$.

${ }^{\mathrm{c}}$ Dose/AUC.

${ }^{\mathrm{d}} V_{\text {central }}\left(K_{34} / K_{43}\right)$

${ }^{\text {e }} V_{\text {central }}+V_{2}$

${ }^{\mathrm{f}} A_{1}\left[\left(1 / K_{13}\right)+\right.$ lag time 1$]+A_{2}\left[\left(1 / K_{23}\right)+\right.$ lag time 2$]$, reference [18].

g $\beta+\alpha=K_{34}+K_{43}+K_{30} ; \beta \alpha=K_{43} K_{30}$.

$\mathrm{h} 0.693 / K$.

${ }^{\mathrm{i}}$ As predicted by NONMEM.

j $1-A_{1}\left(0.95-A_{1}\right.$ for the sustained release formulation).

\section{Results and Discussion}

Fitted and derived population parameters are summarized in Tables 1 and 2. The micro-rate constants $\left(K_{34}\right.$ and $\left.K_{43}\right)$ and volume of distribution $\left(V_{\text {central }} / f\right)$, where $\mathrm{F}$ is the absolute bioavailability, are estimated by NONMEM without I.V. data due to the simultaneous data fitting of the three forms and the assumption that disposition is unchanged between dosage forms. The population serum level profiles (Figure 2) are determined by NONMEM. The population absorption profiles (Figure 3) are characterized using the program TOPFIT [18]. We noted essentially complete absorption $(\geq 95 \%)$ within about $7.5 \mathrm{~h}$ after the enteric-coated formulation and essentially complete absorption after the suppository formulation within about $4.5 \mathrm{~h}$. However, absorption lasts for up to $24 \mathrm{~h}$ in the sustained-release formulation, suggesting absorption of the drug from the colon during its transit through the large intestine. The sustained-release product shows a flip-flop model of slow first-order release in vivo because alpha and beta disposition constants are greater than $K_{13}$ and $K_{23}$ in this formulation.

For the enteric-coated conventional tablet, little or no release takes place in the acidic medium of the stomach. However, as the drug leaves the stomach and enters the small intestine, it is subjected to the intestinal fluids of $\mathrm{pH}$ 5.5-6.8. At this $\mathrm{pH}$, the enteric coat commences to expose the drug to the action of the intestinal $\mathrm{pH}$ in which the solubility of diclofenac sodium is fairly high, which results in high dissolution and hence higher absorption into the blood stream. The release from enteric-coated tablet can have a large variability since it is affected by the weak acid polymer $p K_{a}$, polymer thickness, diclofenac $\mathrm{pK}_{\mathrm{a}}$, diclofenac concentration, and $\mathrm{pH}$ at the site of absorption. The apparent double-peak phenomenon in the population serum level profile (which is not observed in individual curves) is due to the large differences in individual $t_{\max }$ values. This phenomenon was reported previously [19] where composite curves were made.

The absorption and serum profiles of the sustained-release formulations can be correlated to the mechanism of drug release from the film-coated matrix tablet. The release from such a formulation is not dependent on $\mathrm{pH}$, enzymes, or drug location in the GIT [20]. The drug is thought to be leached out by a slow first-order diffusion process through the homogeneous matrix (ethylcellulose derivative) into the GIT fluid [20,21]. The insoluble polymer shell is excreted with little change in its shape. The film coating is not acid resistant and hence no lag time is observed. Then a gradual release through the homogenous matrix occurs slowly throughout the drug intestinal residence time.

The relative bioavailabilities of diclofenac sodium sustained-release and suppository formulations compared to the immediate-release formulations are $90-99 \%$ and $55 \%$ respectively as shown in Table 2 . It is worthwhile noting that fractional absorption for the sustained-release formulation during the first 10 $\mathrm{h}$ is $70-75 \%$, so about $25-30 \%$ of the drug is absorbed from the large intestine.

For the suppository formulation, absorption is essentially complete ( $\geq 95 \%$ ) within $4.5 \mathrm{~h}$ as shown in Figure 3. However, it is only 55\% bioavailable compared to the immediate-release product. This may be due to the low colonic surface area as well as reduced residence time in the colon due to induced defecation by the suppository matrix (synthetic fats). This suggests that the suppository formulation may not be suitable for many patients due to psychological and/or physiological factors. However, the onset of action is faster than the enteric-coated formulation because a gastric emptying lag time prior to absorption is not a factor. The release from the suppository base follows apparent first-order kinetics and the rectal absorption of drug is suggested to be by simple diffusion of the ionized lipophilic (high diffusion in the unstirred aqueous layer and high membrane permeability) molecules in the rectum, $\mathrm{pH} \cong 7$, where the drug partition coefficient is around 14. In addition, the mucosal irritation to the rectum caused by the drug can increase drug permeation [12]. 




Figure 2. Population serum level profiles for the enteric-coated oral formulation, the sustained-release oral formulation, and the suppository formulation of diclofenac sodium after a $100 \mathrm{mg}$ dose

As shown in Table 1, a high inter-individual variability exists in the absorption (release) rate of the enteric-coated form due to $\mathrm{pH}$-dependent solubility of diclofenac sodium throughout the small intestine, $\mathrm{pH}$-dependent dissolution of the enteric coating polymer, and possible variability in coating thickness. The high inter-individual variability in the lag time of the enteric-coated form is due to gastric emptying differences. However, little variability exists in the disposition parameter $K_{34}$, which supports the assumption of the similarity of disposition among all forms. Also, little variability is observed in the absorption rate from the suppository since $\mathrm{pH}$ is fairly stable in the colon. On the other hand, there is high inter-individual variability in the fraction absorbed, $A_{1}$, from the suppository due to variability in colonic residence times.

\section{Conclusions}

Diclofenac sodium population absorption and disposition parameters were determined by simultaneous data fitting using NONMEM. The sustained release from the matrix in vivo is a slow first-order process that is independent of $\mathrm{pH}$, gastric emptying, and GI motility. However, diclofenac sodium is more highly soluble at basic $\mathrm{pH}$ (about 4000 times) as compared to acidic $\mathrm{pH}$. This explains the double peaks in the plasma profile of the sustained-release formulation. The absorption of nonionized drug from the sustained release in the stomach is decreased by drug precipitation, which is responsible for the smaller first peak. Then, once the drug is emptied to the intestine, continuous release and direct absorption takes place over $24 \mathrm{~h}$ throughout the rest of the GIT. This suggests absorption of the drug from the colon takes place since the drug is highly lipophilic. Overall, its relative bioavailability as compared to the enteric-coated formulation is $90-99 \%$, suggesting its suitability as an alternative formulation to the enteric-coated product. Only 70$75 \%$ of the sustained release bioavailable dose is absorbed within the first $10 \mathrm{~h}$. This suggests that for a drug to have good bioavailability as a sustainedrelease formulation, it should have good permeability and lipophilicity so that it is absorbed throughout the small intestine and colon.

The enteric-coated formulation is absorbed rapidly and eliminated rapidly which explains the necessity of the sustained-release formulation. Its release mechanism arises from drug leaching through the dissolving enteric coat as the tablet enters the basic intestinal $\mathrm{pH}$. Then, complete disintegration takes place causing continuous and essentially complete absorption throughout the small intestine. The double-peak phenomenon is due to large differences in individual $t_{\max }$ values.

The suppository formulation provides advantages of faster onset of action due to higher permeability in the colon, and of lower gastric irritation to patients with gastric sensitivity to NSAIDs. The high permeation may be due to the local irritation of the rectal mucosa by the drug and/or the drug's high passive diffusion since its concentration is high in the rectum compared to the small intestine. However, it is only $55 \%$ bioavailable compared to the enteric-coated oral formulation, which may be due to incomplete absorption, since the suppository base induces defecation that results in removal of the drug product prior to complete absorption. This suggests that the suppository formulation is not suitable for many patients.

\section{Acknowledgements}

We thank Dr. E. Lipka for her advice about the NONMEM control file, and Dr. S. Olson for the theoretical interpretation of some points $\mathrm{n}$ the NONMEM program. This work was supported by 


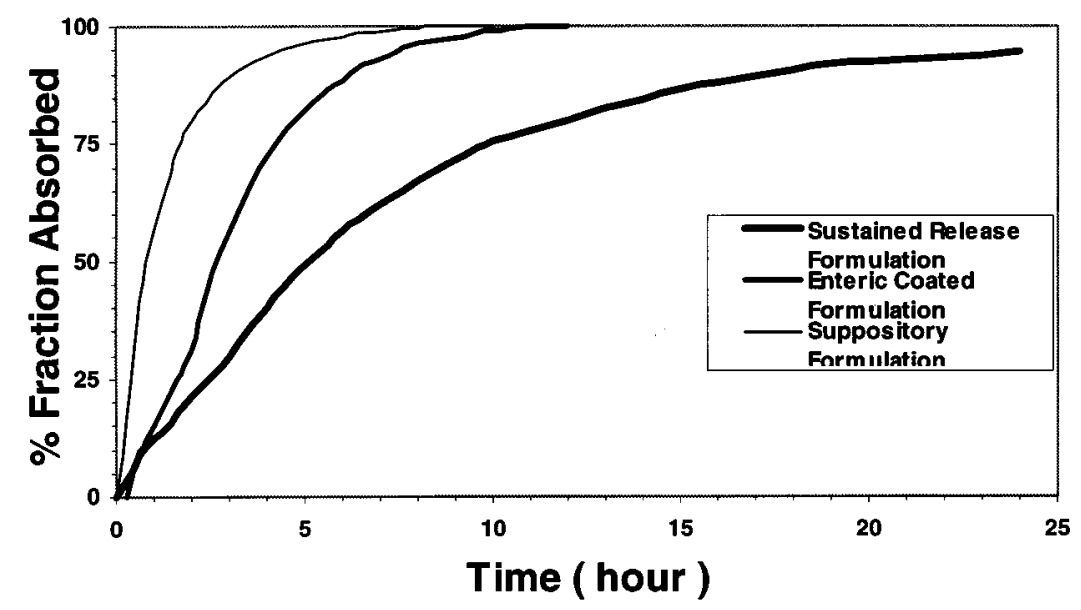

Figure 3. Population absorption profiles for the enteric-coated oral formulation, the sustained-release oral formulation, and the suppository formulation of diclofenac sodium after a $100 \mathrm{mg}$ dose

\section{the Jordan University of Science and Technology} and the FDA.

\section{References}

1. R. Menasse, P.R. Hedwall, J. Kraetz, C. Pericin, L. Riesterer, A. Sallmann, R. Ziel and R. Jaques, Pharmacological properties of diclofenac sodium and its metabolites. Scand. J. Rheumatol. Suppl., 22, 5-16 (1978).

2. L. Solomon and G. Abrams, Voltaren in the treatment of rheumatoid arthritis. S. Afr. Med. J., 48, 949-952 (1974).

3. L.O. Rebecca, D. Helena, L.W. Shekman, K.H.C. Heith and J.S. Ronald, Pharmacokinetics and metabolism of diclofenac sodium Yucatan miniature pigs. Pharm. Res., 11(5), 698-703 (1994).

4. L.L. Leonard, L. Charles and P.G. Morton, Drug Information Handbook, 1994-1995, Lexi-Comp, Hudson, OH, 1994.

5. J.V. Willis, M.J. Kendall, R.M. Flinn, D.P. Thornhill and P.G. Welling, The pharmacokinetics of diclofenac sodium following intravenous and oral administration. Eur. J. Clin. Pharmacol., 16, 405-410 (1979).

6. H.S.H. Lau, K. Chan, L.Y. Shum, S. Adair, H. Ross, H. Eyring, D. Guase and V. John, Dose-proportionality of diclofenac sodium in man. Pharm. Res., 6, S-194 (1989).

7. J.V. Willis and M.J. Kendall, Pharmacokinetic studies on diclofenac sodium in young and old volunteers. Scand. J. Rheumatol. Suppl., 22, 36-41 (1978).

8. E. Perucca, R. Grimaldi, G. Gatti, M. Caravaggi, F. Crema, S. Leccbini and G.M. Frigo, Pharmacokinetic and pharmacodynamic studies with a new controlled-release formulation of propranolol in normal volunteers: a comparison with other commercially available formulations. Br. J. Clin. Pharmacol., 18, 37-43 (1984).

9. M.R. Marino, M. Dey, D.C. Garg, N.S. Jalland, D.M. Dorick, J.J. Martinez and D.J. Weidler, Pharmacokinetics and pharmacodynamics of long-acting propranolol 60-mg capsules: a comparative evaluation. J. Clin. Pharmacol., 27, 885-891 (1987).

10. W. Reiss, H. Stierlin, J.W. Faigle, V.P. Gerardin, K.Schmid, M.
Sulc, J. Wagner and W.P. Theobold, Kinetik und biotransformation von diclofenac in tier und mesch. Therapiewoche, 26, 18-27 (1976).

11. H. Mascher, The pharmacokinetics of a new sustained release form of diclofenac sodium in humans. Drug Des. Dev., 4, 303-311 (1989).

12. Y. Hideya, N. Tohru, T. Harumasa, M. Teruo, Y. Masaru, K. Akira and S. Hajime, Rectal delivery of antiinflammatory drugs; the influence of basic amino acid salts on rectal absorption of diclofenac. Chem. Pharm. Bull., 29, 3326-3333 (1981).

13. M.S. Suleiman, N. Najib, Y. El-Sayed, M. Hassan and M. Abdulhameed, A study on the relative bioavailability of a sustained release formulation of diclofenac sodium. Int. J. Clin. Pharmacol. Ther. Toxicol., 27, 276-279 (1989).

14. Y. El-Sayed, M. Suleiman, M. Hassan, N. Najib, H. Muti and M. Abdulhameed, Comparative bioavailability and in vitro characterization of two brands of diclofenac sodium entericcoated tablets. Inter. J. Clin. Pharmacol. Ther. Toxicol., 26, 487-491 (1988).

15. Y.M. El-Sayed, M.E. Abdulhameed, M.S. Suleiman, N.M. Najib, A rapid and sensitive high-performance liquid chromatographic method for the determination of diclofenac sodium in serum and its use in pharmacokinetics studies. $J$. Pharm. Pharmacol., 40, 727-729 (1988).

16. Data of the human studies done at Jordan University of Science and Technology and Amman Islamic Hospital, provided by Dr. Naji M. Najib.

17. A.J. Boeckmann, L.B. Sheiner and S.L. Beal, NONMEM Manuals, University of California at San Francisco, 1992.

18. G. Heinzel, R. Woloszczak and P. Thomann, TOPFIT manual. In Pharmacokinetic and Pharmacodynamic Data Analysis System for the PC, Version 2, 1993.

19. M.J. Kendall, D.P. Thornhill and J.V. Willis, Factors affecting the pharmacokinetics of diclofenac sodium (Voltarol). Rheumatol. Rehabilitation, Suppl. 2, 38-46 (1979).

20. M.A. Hamed, Dissolution, Bioavailability and Bioequivalence, Mack, Chapters 12 and 23, 1989.

21. A.R. Pena, M. Poncet, J.C. Jinot and D. Chulia, Statistical optimization for a sustained-release preparation with a diclofenac sodium base in inert matrices, II. Pharma Acta Helv., 63, 333-342 1988. 